\title{
Real-time measurements of diffraction grating growth in photopolymer recording materials
}

\author{
Milan Květoň ${ }^{1, a}$, Antonín Havránek ${ }^{2}$, Marek Škereň ${ }^{1}$, and Pavel Fiala ${ }^{1}$ \\ 1 Department of Physical Electronics, Faculty of Nuclear Sciences and Physical Engineering, Czech Technical University \\ in Prague, 11519 Prague 1, Czech Republic \\ 2 Department of Macromolecular Physics, Faculty of Mathematics and Physics, Charles University, 18000 Prague 8, \\ Czech Republic
}

\begin{abstract}
Self-developing ability of photopolymer recording material enables measurements of growth of a refractive index grating in the real-time during a holographic exposure. In our laboratory, we prepare thin layers of photopolymer recording materials and measure their response on harmonic interference field. The results of the measurements are used for characterization of the recording process running in photopolymers and optimization of their chemical composition. Recently, the detection setup has been adapted to measure the phase shift between the recording interference field and the refractive index grating simultaneously with the grow-curves. The phase shift measurement is especially important in the case of photopolymers with nanoparticles as they are spatially redistributed within the layer during the holographic exposure. In the paper, we introduce the principles of the measurement technique, describe our exposure and detection setup, analyze the grow-curve, and show some results obtained from our measurements of the newly developed photopolymer with $\mathrm{AgBr}$ nanoparticles.
\end{abstract}

\section{Introduction}

Photopolymers are attractive recording materials for different holographic applications such as display holography, holographic optical elements, or holographic interferometry. In comparison with silver-halide emulsions or dichromated gelatin (classic holographic recording media), a volume phase grating is formed already during the holographic exposure and no additional wet chemical developing process is needed. Hence, the self-developing ability enables measurements of growth of a refractive index grating in the real-time during the exposure. In our laboratory, we prepare thin layers of photopolymer recording materials and measure their response on harmonic interference field. We have already prepared and tested acrylamide based material with polyvinyl alcohol binder, epoxy matrix, or nanoparticles. Some of our results obtained on acrylamide based photopolymers were published in [1].

The principle of the detection method consists in the real-time measurement of a diffraction efficiency of a forming grating. The method is relatively well known (e.g. [2]. A grow-curve is the typical result of the measurement. It has several phases and analyzing its course, parameters such as initiation time, optimal exposure dose, maximum refractive index modulation, or some polymerization rate constants can be obtained. Very recently, we have adapted the detection setup to measure the phase shift between the recording interference field and the refractive index grating simultaneously with the grow-curves. The phase shift measurement is especially important in the case of photopolymers with nanoparticles as they are spatially redistributed within the layer during the holographic exposure. The mechanisms of spatial exclusion of nanoparticles is not very

\footnotetext{
a e-mail: milan.kveton@fjfi.cvut.cz
}

clear and the phase-shift measurement help for better understanding of it.

In the paper, the mechanism of polymer recording will be shortly introduced, the preparation procedure and chemical composition of our newly developed photopolymer with $\mathrm{AgBr}$ nanoparticles will be given, the principles of the detection methods will be described, and results of our measurements will be presented and discussed.

\section{Photopolymer recording medium}

Photopolymer recording materials are known for more then 40 years. They are typically composed of molecules of initiators and monomers which polymerize after illumination of light. In last 10 years, photopolymerizable recording materials with nanoparticles are the subject of an intensive study [3-7]. In comparison with common photopolymers, they contain inert inorganic particles which have special optical, magnetic, or luminescent properties. Similarly as in the case of common photopolymers, the materials are self-developing and no additional wet chemical developing process is needed. After a holographic exposure, a periodic spatial distribution of nanoparticles is formed through the inhomogeneous polymerization process. The formed structure in the composite system combines special properties of nanoparticles and diffractive properties of their periodic distribution. In the case of a recording material for optical holography, the photopolymerizable system includes nanoparticles with a refractive index which differs from a host matrix. $\mathrm{TiO}_{2}, \mathrm{SiO}_{2}$, or $\mathrm{ZrO}_{2}$ nanoparticles are used most often and their redistribution during a holographic exposure produces efficient diffraction gratings. In our case, $\mathrm{AgBr}$ nanoparticles are embedded in a gelatin layer as the refractive index difference is relatively high (2.3 vs 1.5$)$.

This is an Open Access article distributed under the terms of the Creative Commons Attribution License 2.0, which permits unrestricted use, distribution, and reproduction in any medium, provided the original work is properly cited. 


\subsection{Mechanism of polymer recording}

In the immobilization-diffusion theory of photopolymer holographic recording $[8,1]$, we described why the growing photopolymer was located in the illuminated parts of the recording medium and why its position was practically fixed after the exposure. Details of the argumentation are thoroughly given in the cited references. The location of the photopolymer remains the same in the recording materials with nanoparticles as it has been in the previously studied acrylamide-based recording materials. However, as the nanoparticles are not compatible with the polymer, they are extruded out of the illuminated parts of the recording material and create an inverse distribution with respect to the interference field and polymer density. In a simple way, the mechanism of polymer recording is described in figure 1. More detailed discussion on the physical chemistry description of the grating growth in composite systems lies beyond the capacity of this short article (some particularities of the problem can be found in [9]).

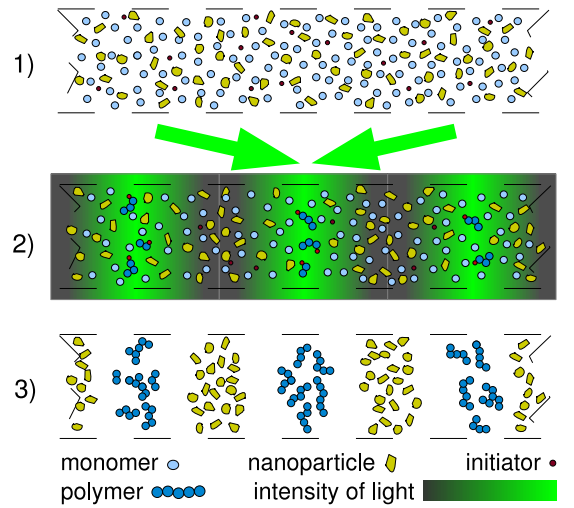

Fig. 1. Formation of a holographic grating in photopolymers distribution of components within the recording layer before 1), during 2), and after 3) a holographic exposure.

\subsection{Material composition an preparation}

The recording material, which is further used in the experiments, is composed of a gelatin binder with $\mathrm{AgBr}$ nanoparticles and acrylic monomers with a photoinitiation system (see table 1). Typically, the diameter of nanoparticles is about $30 \mathrm{~nm}$ and they have relatively narrow size distribution. The preparation procedure of a recording layer consists of several steps which involve controlled precipitation of $\mathrm{AgBr}$ grains in gelatin solution, addition of a photopolymerization system, and coating thin layers. The prepared layers are typically $20 \mu \mathrm{m}$ thick and are deposited on a glass plates. The absorption maximum of the material is at a $550 \mathrm{~nm}$ wavelength (green light), its optimal exposure dose ranges from 5 to $10 \mathrm{~mJ} / \mathrm{cm}^{2}$, and the diffraction efficiency of a formed grating (volume phase transmission grating) reaches $80 \%$.
Table 1. Chemical composition of the photopolymer recording material with $\mathrm{AgBr}$ nanoparticles dispersed in a gelatin matrix.

\begin{tabular}{llr}
\hline Chemical & function & weight fraction \\
\hline Gelatin & matrix & $20 \%$ \\
AgBr & nanoparticle & $15 \%$ \\
Acrylic acid & liquid monomer & $57 \%$ \\
N,N-Dimethylacrylamide & liquid monomer & $7 \%$ \\
H-Nu 535 & photoinitiator & $0.2 \%$ \\
Borate V & co-initiator & $0.8 \%$ \\
\hline
\end{tabular}

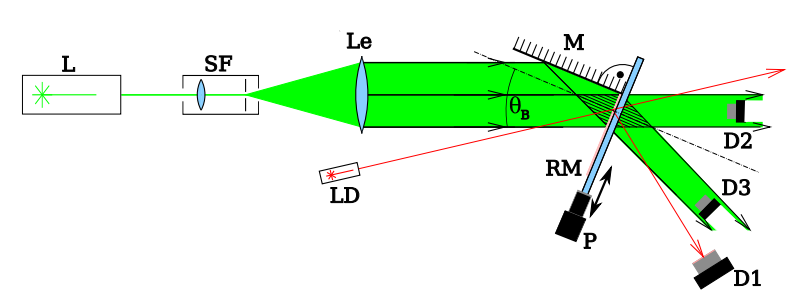

Fig. 2. Schema of the experimental setup. Legend: L - recording laser (532 nm), SF - spatial filter, Le - collimating lens, M - plane mirror, RM - recording material, LD - laser diode $(656 \mathrm{~nm}), \mathrm{D} 1$, D2, D3 - photo-detectors, $\mathrm{P}$ - piezoelectric actuator.

\section{Real-time characterization}

\subsection{Detection of a diffraction grating growth}

With the detection method, a response of the recording material on exposure of the harmonic interference field is tested. A volume phase diffraction grating is formed already during the exposure and its diffraction efficiency is continuously measured. The diffraction grating is characterized with the diffraction efficiency $\eta$, but the main quantity of the recording material is the refractive index modulation $n_{1}$. It can be evaluated from the measurement of the diffraction efficiency with Eq. (1) derived in the coupled wave theory [10]. The material parameter refractive index is connected with the chemical composition of the recording material. So measured changes of the refractive index may also be used for characterization of recording processes (polymerization, diffusion) and their further optimization.

In figure 2, a schema of the recording and detection setup is given. With the setup, an unslanted transmission grating is recorded with two "s" polarized waves. For the exposure, the diode-pumped, frequency-doubled Nd:YAG laser, that emits at a $532 \mathrm{~nm}$ wavelength, is used. A narrow beam of the detection laser diode, which wavelength is out of the absorption band of the material, is used for the measurement of the diffraction efficiency. The beam is adjusted at the Bragg angle of the recorded grating. The length of the exposure is controlled with an electronic shutter which also triggers the real-time measurement of $\eta(t)$. The refractive index modulation $n_{1}(t)$ is evaluated as an inverse function from the expression which was derived in the coupled wave theory [10]

$$
\eta(t)=\sin ^{2} \frac{\pi n_{1}(t) d}{\lambda_{r} \cos \theta_{B}},
$$

where $d$ is the thickness of a recording layer, $\lambda_{r}$ is the reconstruction wavelength and $\theta_{B}$ is the Bragg angle. The obtained dependence of the refractive index modulation 
on time we call the grow-curve and is used for characterization of self-developing recording materials. More details about the detection method and experimental setup can be found in our recent paper [1] which deals with the acrylamide-based photopolymer.

\subsection{Phase shift measurements}

From the previously described measurement, only the amplitude $n_{1}$ of the refractive index distribution $n(x)=n_{0}+$ $n_{1} \cos (K x)$ is obtained. In photopolymers, due to the effect of wave mixing and the presence of nanoparticles, the forming grating can be phase shifted with respect to the recording interference field. The phase shift was theoretically analyzed in our paper [11]. For the transmitted intensities $I_{1}(d)$ and $I_{2}(d)$ behind the forming grating of the thickness $d$, we derived following expressions:

$$
\begin{aligned}
& I_{1}=I_{10} \cos ^{2} \kappa d+I_{20} \sin ^{2} \kappa d-\sqrt{I_{10} I_{20}} \sin 2 \kappa d \sin \varphi, \\
& I_{2}=I_{10} \sin ^{2} \kappa d+I_{20} \cos ^{2} \kappa d+\sqrt{I_{10} I_{20}} \sin 2 \kappa d \sin \varphi,
\end{aligned}
$$

where $I_{10}$ and $I_{20}$ are incident intensities, $\kappa$ is the coupling constant and $\varphi$ is the phase shift between the grating and the interference field. The coupling constant $\kappa=$ $\pi n_{1} /(\lambda \cos \Theta)$ and it can be evaluated if $n_{1}$ is substituted from the grow-curve measurements. From expressions (2) and (3) follows that transfer of energies between waves occurs if $I_{1}$ and $I_{2}$ differ or the the phase $\varphi$ is nonzero. Hence, from the real-time measurements of $I_{1}$ and $I_{2}$ behind the grating, the phase can be obtained. In our setup in figure 2, intensities of transmitted waves are measured with detectors D2 and D3.

\section{Results}

The process of a grating formation was measured with the method described in the previous section. Typical growcurves obtained from the real-time measurement are presented in figure 3 and figure 4 . The grow-curve describes the process of a diffraction grating formation through the parameter $n_{1}$. At the beginning of the exposure, a short induction period is observed. In spite of no detectable change of the refractive index, some initiation processes may be running in the recording material. After the induction period, the growth of the grating begins. Typically, the formation rate increases in the beginning of this phase. The grow-curve goes through its inflexion point and the formation rate decreases at the end of the exposure phase. The length of the induction period and the rate of a grating formation depends on exposure parameters and chemical composition of the material.

\subsection{Influence of exposure parameters}

An influence of the recording intensities was measured at first and the results are given in figure 3 . In the case of a higher recording intensity, a higher recording rate is detected than that in the case of a lower intensity. The effect of intensity can be theoretically explained by the fact that the polymerization rate increases with increasing intensity

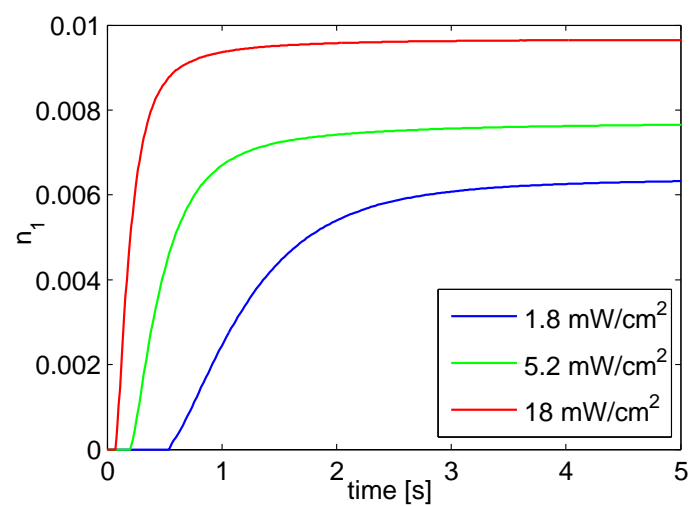

Fig. 3. Influence of recording intensities on the process of a grating formation; exposure parameters $\Lambda=700 \mathrm{~nm}$ and $t_{e}=5 \mathrm{~s}$.

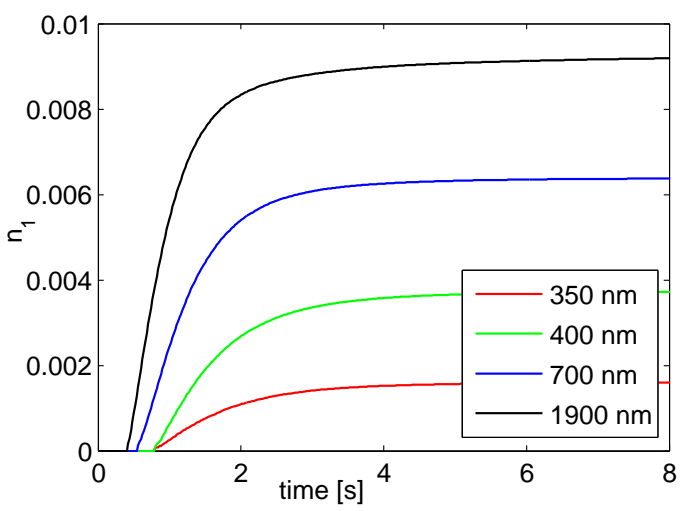

Fig. 4. Influence of spatial period $\Lambda$ on the process of a grating formation; exposure parameters $I_{1}=I_{2}=1.8 \mathrm{~mW} / \mathrm{cm}^{2}$ and $t_{e}=$ $8 \mathrm{~s}$.

of light as more radicals are produced. The polymerization process is assumed as the driving mechanism and so the higher polymerization rate causes faster formation of a grating. Next to the rate of the grating growth, the final value of the refractive index modulation depends also on the recording intensity. This effect is still not very clear although it has been observed in other types of photopolymer recording materials.

The grating growth depends strongly on the spatial period of the recording interference field (see figure 4). In the case of a long period, the grating grows up rapidly and a high value of $n_{1}$ is reached. On the other hand, a short spatial period is difficult to record and a low value of $n_{1}$ is reached. We assume that this effect of the limited resolution is caused by the size and mobility of the growing polymers. Hence their positions are not located only in bright areas of the interference field, which are very narrow in this case, and the separation process of nanoparticles and polymers does not proceed optimally.

\subsection{SEM observations}

The direct observations with a scanning electron microscope (SEM) give evidences of redistribution of nanoparticles within the layer. With SEM, only $\mathrm{AgBr}$ nanoparticles can be observed; the other components such as monomer, polymers, or gelatin matrix are not visible. Before a holographic exposure, the distribution of nanoparticles in an 


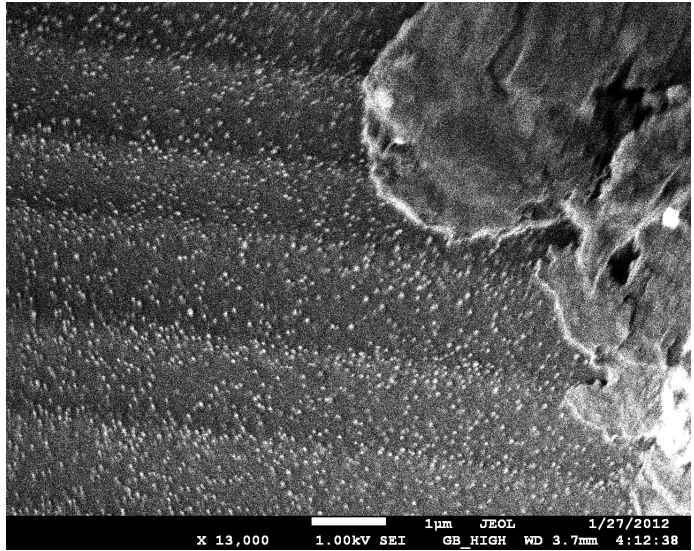

Fig. 5. SEM images of the cross section of a grating recorded in photopolymer with $\mathrm{AgBr}$ nanoparticles.

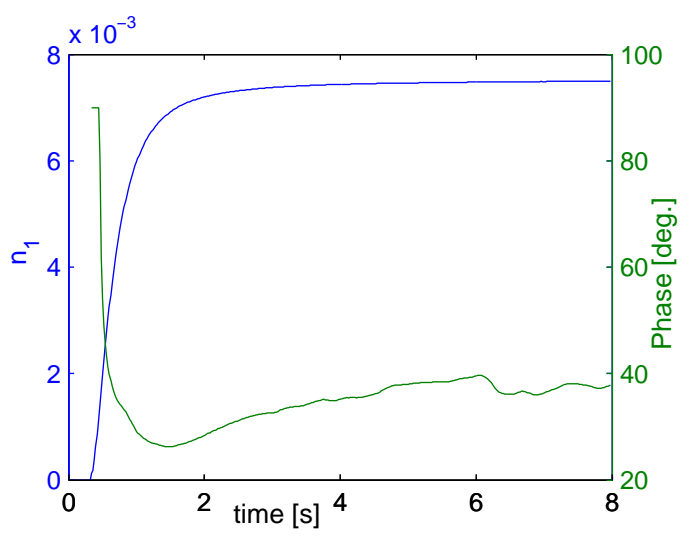

Fig. 6. Grow-curve and time development of the phase shift during the recording process. Exposure parameters were $I_{1}=I_{2}=$ $5.2 \mathrm{~mW} / \mathrm{cm}^{2}, \Lambda=700 \mathrm{~nm}$, and $t_{e}=8 \mathrm{~s}$.

unexposed material is homogeneous. SEM image of a cross section of the exposed material (grating) in the thickness direction are shown in figure 5 . In the figure, a periodic spatial distribution of nanoparticles can be seen.

\subsection{Phase shift measurement}

From SEM observations, we are not able to determine the phase shift between the interference field and grating. So, we have applied the method based on measurements of transmitted intensities of the recording waves. In figure 6 , the grow-curve and the phase are plotted together. Despite the fact that both intensities of incident recording waves were the same, a phase shift between the field and grating exists from the very beginning of the grating growth. With the measurement, we can not give an evidence that the distribution of nanoparticles is inverse or not with respect to the field as the evaluated phase shift is in the interval from -90 to 90 degrees.

The influence of the phase shift on the recording process will be further studied. We also want to apply an additional phase shift of the grating with a piezoelectric actuator (see setup in figure 2) to prove that the refractive index grating produced with $\mathrm{AgBr}$ nanoparticles produce an inverse distribution of the refractive index with respect to the recording field. Therefore, we will follow the method described in [12].

\section{Conclusions}

We have describe the real-time measurement of diffraction grating growth in photopolymerizable recording systems. The standard detection technique has been recently adapted to measure the phase shift between the recording interference field and the refractive index grating. With the real-time detection method, the photopolymer with $\mathrm{AgBr}$ nanoparticles has been characterized. It has been found that high values of the index modulation are reached for broader spatial periods and higher recording intensities. The evidence, that the grating is produced due to redistribution of nanoparticles during a holographic exposure, has been given from SEM observations of the grating microstructure. Our preliminary results of the phase shift measurement show that a phase shift between the field and grating can exists even if both recording intensities are similar.

\section{Acknowledgments}

This work was supported by the Grant Agency of Academy of Sciences of the Czech Republic under the project KAN 401220801.

\section{References}

1. M. Kveton, P. Fiala, A. Havranek, in Holography, Research and Technologies, edited by J. Rosen (Intech, 2011), pp. $58-82$

2. S. Martin, P. Leclere, Y. Renotte, V. Toal, Y. Lion, Optical Engineering 33, 3942 (1994)

3. N. Suzuki, Y. Tomita, Appl. Optics 43, 2125 (2004)

4. Y. Tomita, N. Suzuki, K. Chikama, Opt. Lett. 30, 839 (2005)

5. A.T. Juhl, J.D. Busbee, J.J. Koval, L.V. Natarajan, V.P. Tondiglia, R.A. Vaia, T.J. Bunning, P.V. Braun, ACS Nano 4, 5953 (2010)

6. I. Naydenova, E. Leite, T. Babeva, N. Pandey, T. Baron, T. Yovcheva, S. Sainov, S. Martin, S. Mintova, V. Toal, J. Phys. 13 (2011)

7. T.N. Smirnova, L.M. Kokhtich, O.V. Sakhno, J. Stumpe, Opt. Spectrosc. 110, 129 (2011)

8. A. Havranek, M. Kveton, J. Havrankova, Pol. Bull. 58, 261 (2007)

9. C.M. Leewis, A.M. de Jong, L.J. van IJzendoorn, D.J. Broer, J. Appl. Phys. 95, 4125 (2004)

10. H. Kogelnik, Bell Syst. Tech. J. 48, 2909 (1969)

11. M. Kveton, I. Richter, P. Fiala, A. Havranek, J. Modern Optics 51, 2241 (2004)

12. N. Suzuki, Y. Tomita, Appl. Phys. Lett. 88 (2006) 